\title{
Evaluating 'elliptic' master integrals at special kinematic values: using differential equations and their solutions via expansions near singular points
}

\author{
Roman N. Lee, ${ }^{a}$ Alexander V. Smirnov ${ }^{b, d}$ and Vladimir A. Smirnov ${ }^{c}$ \\ ${ }^{a}$ Budker Institute of Nuclear Physics, \\ 630090 Novosibirsk, Russia \\ ${ }^{b}$ Research Computing Center, Moscow State University, \\ 119992 Moscow, Russia \\ ${ }^{c}$ Skobeltsyn Institute of Nuclear Physics of Moscow State University, \\ 119992 Moscow, Russia \\ ${ }^{d}$ Institut für Theoretische Teilchenphysik, KIT, \\ 76128 Karlsruhe, Germany \\ E-mail: roman.n.lee@gmail.com, asmirnov80@gmail.com, \\ smirnov@theory.sinp.msu.ru
}

ABSTRACT: This is a sequel of our previous paper where we described an algorithm to find a solution of differential equations for master integrals in the form of an $\epsilon$-expansion series with numerical coefficients. The algorithm is based on using generalized power series expansions near singular points of the differential system, solving difference equations for the corresponding coefficients in these expansions and using matching to connect series expansions at two neighboring points. Here we use our algorithm and the corresponding code for our example of four-loop generalized sunset diagrams with three massive and two massless propagators, in order to obtain new analytical results. We analytically evaluate the master integrals at threshold, $p^{2}=9 \mathrm{~m}^{2}$, in an expansion in $\epsilon$ up to $\epsilon^{1}$. With the help of our code, we obtain numerical results for the threshold master integrals in an $\epsilon$-expansion with the accuracy of 6000 digits and then use the PSLQ algorithm to arrive at analytical values. Our basis of constants is build from bases of multiple polylogarithm values at sixth roots of unity.

KeYwords: Perturbative QCD, Scattering Amplitudes

ArXiv EPrint: 1805.00227 


\section{Contents}

1 Introduction 1

$\begin{array}{lll}2 & \text { Setup } & 2\end{array}$

3 Four-loop generalized sunset diagram at threshold $\quad 4$

4 Conclusion $\quad 12$

\section{Introduction}

Analytical results for Feynman integrals can, typically, be expressed in terms of harmonic polylogarithms [1] or multiple polylogarithms [2] which are very well mathematically studied special functions. For harmonic polylogarithms, one can apply the package HPL [3] which encodes various analytical properties and provides the possibility of numerical evaluation with a desirable precision. For multiple polylogarithms, one can either use the computer code [4] based on the GiNaC library [5] to obtain high-precision numerical values or construct a code based on their algebraic properties, for example, to reveal their behavior near singular points. Anyway, with a result in terms of these functions at hand, one can evaluate a Feynman integral at regular points and obtain expansions at singular points.

The possibility to arrive at a result written in terms of these functions exists if, within the method of differential equations [6-11], one succeeds to turn to a so-called canonical basis [12] of the master integrals. ${ }^{1}$ It is well known that the $\epsilon$-form of DE for a given set of the master integrals can not always be achieved with rational, or even algebraic transformations. $^{2}$ The simplest counter example is given by the two-loop propagator sunset diagram with three identical masses. In many irreducible cases, the lowest nontrivial term of $\epsilon$-expansion is expressed in terms of elliptic integrals, and in what follows we will also refer to these cases as 'elliptic' in no relation with the functional form of the coefficients of $\epsilon$ expansion.

In situations without canonical bases, one can hope that the number of elliptic master integrals is small and try to obtain, in these cases, two and three-fold parametric representations suitable for numerical evaluation, and in all other cases to proceed with canonical subbases - see examples of such an approach in refs. [20-23]. On the other hand, it is quite natural to try to introduce new functions which would enable us to present results, in elliptic cases, in an analytical form. Multiple suggestions to introduce elliptic generalizations of multiple polylogarithms can be found in refs. [24-32]. However, these new functions do not have the same status as harmonic and multiple polylogarithms, as far as a

\footnotetext{
${ }^{1}$ There are various codes to arrive at a canonical form (or, $\epsilon$-form [13]) — see [13-18].

${ }^{2}$ Recently, a strict criterion of the existence of an $\epsilon$-form was presented in ref. [19].
} 
detailed description of their properties and the possibility to evaluate them numerically are concerned. Moreover, the examples of successful treatment of $\epsilon$-expansion in elliptic cases are, at most at the two-loop level. Anyway, we are very far, even in lower loops orders, from obtaining a complete description of a class of functions which can appear in results for Feynman integrals.

We advocated [33] an alternative way to solve differential equations which can be used also in 'elliptic' situations and illustrated it through a four-loop example. We considered multiloop Feynman integrals depending on one variable, i.e. with two scales where the variable is introduced as the ratio of these scales. We described an algorithm to find a solution of a given differential system in the form of an $\epsilon$-expansion series with numerical coefficients. It is based on using generalized power series expansions near singular points of the differential system, solving difference equations for the corresponding coefficients in these expansions and using matching to connect series expansions at two neighbouring points. We provided a computer code where this algorithm is implemented for a simple example of a family of four-loop Feynman integrals where the $\epsilon$-form is impossible. Using this code it is possible to evaluate master integrals at a given point as well as expansions at singular points with a required precision in an $\epsilon$-expansion with a required number of terms.

Our present paper is a sequel of ref. [33]. The goal here is to apply our algorithm which is numerical in its character and the corresponding code in our example, i.e. fourloop generalized sunset diagrams with three massive and two massless propagators, in order to obtain new analytical results. We analytically evaluate the master integrals at threshold, $p^{2}=9 m^{2}$, in an expansion in $\epsilon$ up to $\epsilon^{1}$. Remember that the $\epsilon$-form of the corresponding equations is impossible so that we cannot use the well-known procedure of using a solution at a general point in terms of well-established special functions and then turn to results at this singular point. However, although solutions to the differential equations look too complicated, the values of the master integrals at some special points can be conventional polylogarithmic constants. We will see that this is indeed true for the $\epsilon$-expansion of the integrals of our family at the threshold. We use our code in order to construct a linear operator (a matrix) which renders the boundary conditions in one, suitable chosen, singular point to the coefficients of asymptotic expansion at the other point, $p^{2}=9 \mathrm{~m}^{2}$ in our case. From these coefficients we extract the values of the integrals at $p^{2}=9 \mathrm{~m}^{2}$.

After reminding the main points of our setup in section 2, we explain in section 3 how we obtain high-precision values, up to 6000 digits, for the threshold integrals and then succeed in finding a relevant basis of constants in order to use the PSLQ algorithm [34]. It turns out that the relevant bases of constants can be constructed starting from the bases of multiple polylogarithm values at sixth roots of unity, i.e. of the form $G\left(a_{1}, \ldots, a_{w} ; 1\right)$ where the indices $a_{i}$ are equal to zero or a sixth root of unity, with $a_{1} \neq 1$, which were constructed in ref. [35] up to weight six. We discuss various perspectives in Conclusion.

\section{Setup}

Differential equations for master integrals have the form

$$
\partial_{x} \boldsymbol{J}=M(x, \epsilon) \boldsymbol{J},
$$


where $x$ is a dimensionless ratio of two scales for a family of dimensionally regularized Feynman integrals depending on two scales, $\boldsymbol{J}$ is a column-vector of $N$ functions, and $M$ is an $N \times N$ matrix with elements which are rational functions of $x$ and $\epsilon$.

The general solution of this linear system has the form

$$
\boldsymbol{J}(x)=U(x) \boldsymbol{J}_{0},
$$

where $\boldsymbol{J}_{0}$ is a column of constants, and $U$ is an evolution operator represented in terms of a path-ordered exponential

$$
U(x)=P \exp \left[\int d x M(x)\right]
$$

We want to expand this operator in the vicinity of each singular point. Without loss of generality, let us consider the expansion near $x=0$. It has the form

$$
U(x)=\sum_{\lambda \in S} x^{\lambda} \sum_{n=0}^{\infty} \sum_{k=0}^{K_{\lambda}} \frac{1}{k !} C(n+\lambda, k) x^{n} \ln ^{k} x,
$$

where $S$ is a finite set of powers, $K_{\lambda} \geqslant 0$ is an integer number corresponding to the maximal power of the logarithm.

We assume that all the singular points of the differential system are regular so that we can reduce the differential system to a local Fuchsian form in any singular point. Therefore, we can reduce it at $x=0$ to normalized Fuchsian form [19] by means of rational transformations. Let us assume that the system is in a global normalized Fuchsian form, i.e.,

$$
M(x)=\frac{A_{0}}{x}+\sum_{k=1}^{s} \frac{A_{k}}{x-x_{k}}
$$

and for any $k=0, \ldots, s$ the matrix $A_{k}$ is free of resonances, i.e. the difference of any two of its distinct eigenvalues is not integer. In particular, the 'elliptic' cases, as a rule, can easily be reduced to a global normalized Fuchsian form.

As it is shown in ref. [33], $S$ and $K_{\lambda}$ can be determined and the difference equations for the coefficients $C(n+\lambda, k)$ in $(2.4)$ can be solved algorithmically. In fact, the idea to use series expansions at singular points and difference equations for the corresponding coefficients is very well known in mathematics. In high-energy physics, this strategy when evaluating Feynman integrals can be found, for example, in refs. [20, 38-41]. Let us emphasize that our algorithm, with the current assumptions, provides solutions with no more than a linear growth of computational complexity with respect to a required number of terms. This is very important for a subsequent matching procedure which enables one to connect series expansions at two neighbouring points and thereby to obtain the possibility to evaluate Feynman integrals at any given point — see details in ref. [33]. 


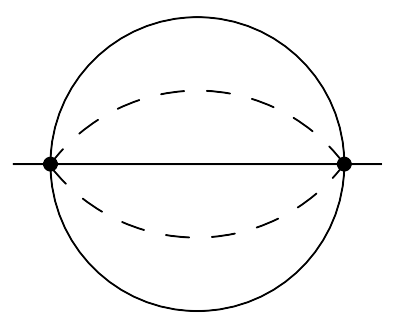

Figure 1. The generalized sunset graph with two massless and three massive lines with the same mass.

\section{Four-loop generalized sunset diagram at threshold}

As in ref. [33] let us consider the example of the following family of four-loop Feynman integrals:

$$
\begin{aligned}
F_{a_{1}, \ldots, a_{14}}=\int \ldots \int & \frac{\mathrm{d}^{D} k_{1} \ldots \mathrm{d}^{D} k_{4}\left(k_{1} \cdot p\right)^{a_{6}}\left(k_{2} \cdot p\right)^{a_{7}}\left(k_{3} \cdot p\right)^{a_{8}}\left(k_{4} \cdot p\right)^{a_{9}}}{\left(-k_{1}^{2}\right)^{a_{1}}\left(-k_{2}^{2}\right)^{a_{2}}\left(m^{2}-k_{3}^{2}\right)^{a_{3}}\left(m^{2}-k_{4}^{2}\right)^{a_{4}}} \\
& \times \frac{\left(k_{1} \cdot k_{2}\right)^{a_{10}}\left(k_{1} \cdot k_{3}\right)^{a_{11}}\left(k_{1} \cdot k_{4}\right)^{a_{12}}\left(k_{2} \cdot k_{3}\right)^{a_{13}}\left(k_{2} \cdot k_{4}\right)^{a_{14}}}{\left(m^{2}-\left(\sum k_{i}+p\right)^{2}\right)^{a_{5}}},
\end{aligned}
$$

where $p$ is the external momentum and $m$ is the mass of three lines. They correspond to the generalized sunset graph shown in figure 1. We introduce $x=p^{2} / m^{2}$.

There are four master integrals in this family. As the primary master integrals we choose the following basis ${ }^{3}$

$$
\left\{F_{1,1,1,1,1,0, \ldots, 0}, F_{1,1,2,1,1,0, \ldots, 0}, F_{1,2,1,1,1,0, \ldots, 0}, F_{1,2,1,1,2,0, \ldots, 0}\right\}
$$

The singular points of the differential equations are $x_{0}=0, x_{1}=1, x_{2}=9$ and $x_{3} \equiv x_{-1}=\infty$. Using our algorithm we presented in ref. [33] the code DESS to evaluate master integrals at a given point as well as expansions at singular points with a required precision keeping a required number of terms in an $\epsilon$-expansion.

Let us now formulate our current goal: to evaluate master integrals of family (3.1) considered at threshold, $p^{2}=9 m^{2}$, i.e. exactly at the singular point $x_{2}=9$. In fact, for such integrals defined with the same general formula (3.1) we have now three master integrals ${ }^{4}$ which can be chosen as

$$
\left\{J_{1}=F_{1,1,1,1,1,0, \ldots, 0}, J_{2}=F_{1,1,2,1,1,0, \ldots, 0}, J_{3}=F_{1,2,1,1,1,0, \ldots, 0}\right\}
$$

This can be done with the code DESS, where boundary conditions at the point $x_{0}=0$ were implemented. This point is, however, not a neighbour of $x_{2}=9$ so that matching is used twice when transporting information between $x_{0}$ and $x_{2}$. This results in the necessity to

\footnotetext{
${ }^{3}$ In our paper, we use FIRE [42-44] in combination with LiteRed [45, 46] to solve integration by parts relations and reveal master integrals.

${ }^{4}$ The decrease of the number of master integrals for special kinematic point is well known. In particular, within the approach of ref. [47], the decrease is related to the fact that one critical level of the polynomial $U+F$ tends to infinity as $s \rightarrow 9$ and also for $s \rightarrow 1$.
} 
evaluate much more terms of the series expansions at the three points $\left\{x_{0}, x_{1}, x_{2}\right\}$ in order to achieve a required precision which, as we will see later, should be very high because of a big number of constants relevant to results at $x_{2}$.

In fact, the choice of the point $x_{0}$ to impose boundary conditions encoded in DESS can be explained by the fact that, generally speaking, setting $p^{2}=0$ for propagator integrals is equivalent to $p=0$ and resulting vacuum Feynman integrals turn out to have just less indices. However, vacuum integrals can involve 'more complicated' constants. To solve our current goal, we can make a better choice to impose boundary conditions at the point $x_{1}$ for two reasons: this is now a neighbour of $x_{2}=9$ and the corresponding constants are multiple zeta values, logarithm of two and polylogarithms of one half. Indeed, master integrals at $x=x_{1}$ appeared in the calculations presented in refs. $[49,50]$ where they were evaluated using a onefold Mellin-Barnes representation. In particular, we have the following result

$$
\begin{aligned}
& \left.F_{1,1,1,1,1,0, \ldots, 0}\right|_{p^{2}=m^{2}=1}=-\frac{1}{4 \epsilon^{4}}-\frac{7}{8 \epsilon^{3}}-\left(\frac{17}{12}+\frac{\pi^{2}}{12}\right) \frac{1}{\epsilon^{2}}+\left(\frac{\zeta(3)}{3}-\frac{835}{576}-\frac{7 \pi^{2}}{24}\right) \frac{1}{\epsilon} \\
& +\left(\frac{7 \zeta(3)}{6}-\frac{7379}{6912}-\frac{17 \pi^{2}}{36}-\frac{3 \pi^{4}}{20}\right) \\
& +\left(\frac{17 \zeta(3)}{9}+\frac{127 \pi^{2} \zeta(3)}{9}-\frac{289 \zeta(5)}{5}+\frac{6766055}{82944}-\frac{41539 \pi^{2}}{1728}-\frac{21 \pi^{4}}{40}\right) \epsilon \\
& +\left(-288 \pi^{2} \operatorname{Li}_{4}\left(\frac{1}{2}\right)-\frac{355325 \zeta(3)}{432}+\frac{889 \pi^{2} \zeta(3)}{18}+\frac{2203 \zeta(3)^{2}}{9}-\frac{2023 \zeta(5)}{10}\right. \\
& -252 \pi^{2} \zeta(3) \log (2)+\frac{1449210865}{995328}-\frac{8822483 \pi^{2}}{20736}-\frac{17 \pi^{4}}{20}+\frac{3877 \pi^{6}}{1890} \\
& \left.-12 \pi^{2} \log ^{4}(2)+12 \pi^{4} \log ^{2}(2)+424 \pi^{2} \log (2)\right) \epsilon^{2} \\
& +(-5760 \zeta(-6,-1)-5760 \zeta(-6,1)-5760 \zeta(-5,2) \\
& -5760 \zeta(-5,1,1)-5760 \zeta(5,-1,-1)+5760 s_{6} \log (2)-10080 \operatorname{Li}_{4}\left(\frac{1}{2}\right) \zeta(3) \\
& +5184 \pi^{2} \operatorname{Li}_{5}\left(\frac{1}{2}\right)-1008 \pi^{2} \operatorname{Li}_{4}\left(\frac{1}{2}\right)-16960 \mathrm{Li}_{4}\left(\frac{1}{2}\right) \\
& +5184 \pi^{2} \operatorname{Li}_{4}\left(\frac{1}{2}\right) \log (2)+\frac{312867 \zeta(7)}{14}-\frac{100204 \pi^{2} \zeta(5)}{15}-\frac{4913 \zeta(5)}{15} \\
& +\frac{15421 \zeta(3)^{2}}{18}+\frac{908 \pi^{4} \zeta(3)}{15}+\frac{2159 \pi^{2} \zeta(3)}{27}-\frac{77124781 \zeta(3)}{5184} \\
& -420 \zeta(3) \log ^{4}(2)+2688 \pi^{2} \zeta(3) \log ^{2}(2)-7200 \zeta(3)^{2} \log (2) \\
& -882 \pi^{2} \zeta(3) \log (2)+\frac{3877 \pi^{6}}{540}+\frac{195233 \pi^{4}}{1728}-\frac{1121725465 \pi^{2}}{248832} \\
& +\frac{182188906799}{11943936}+\frac{864}{5} \pi^{2} \log ^{5}(2)-42 \pi^{2} \log ^{4}(2)-\frac{2120 \log ^{4}(2)}{3} \\
& -144 \pi^{4} \log ^{3}(2)+42 \pi^{4} \log ^{2}(2)-\frac{9328}{3} \pi^{2} \log ^{2}(2)-\frac{14}{3} \pi^{6} \log (2) \\
& \left.+7652 \pi^{2} \log (2)\right) \epsilon^{3}+O\left(\epsilon^{4}\right),
\end{aligned}
$$


where $\zeta(\ldots)$ are multiple zeta values. Here the result is restricted to contributions of weight seven and some efforts are needed to go further.

It turns out that the best way to impose boundary conditions is to choose $x_{3}=\infty$ because the corresponding expansion is nothing but the large-momentum expansion [51-54] where, for our integrals (3.1), any term is a product of one-loop tadpoles and massless propagator integrals and can be evaluated via gamma functions at general $\epsilon$. This provides any required accuracy and any required number of terms in $\epsilon$-expansions in the boundary conditions. In the updated version of our code DESS, we introduce the possibility to impose boundary conditions at an arbitrary singular point. We added one more argument ns to the function

$$
\text { DESS }[\text { rdatas, } x, f(x), o e, n p, n t, n s]
$$

which means the number of a singular point and this number is 1 for $x_{0}, 2$ for $x_{1}$, and 4 for $x_{3}$. There is no sense to choose $x_{2}$ since this point is most complicated from the calculational point of view. We attach also two more auxiliary files: BoundaryConditions1 and BoundaryConditionsInf where analytic results for the boundary integrals are encoded. As before, the code and the auxiliary data can be downloaded from https://bitbucket.org/feynmanintegrals/dess. With the current version of DESS, we have obtained numerical results for the threshold master integrals in an $\epsilon$-expansion up to $\epsilon^{2}$ with the accuracy of 6000 digits for the corresponding coefficients. This took less than four hours on a desktop. As we will see shortly, such a big accuracy is needed for an application of the PSLQ algorithm.

The crucial point is a choice of a relevant basis of constants. A first hint comes from the known results for the two-loop sunset diagram at threshold $[55,56]$ where one can observe multiple polylogarithm values at sixth roots of unity and $\frac{\pi}{\sqrt{3}}$. Let us also take into account that, at least according to refs. [57-59], it might be reasonable to include into the basis the constant $\sqrt{3}$ separately. Therefore, we tried to use the bases connected with multiple polylogarithm values at sixth roots of unity and constructed in ref. [35] up to weight $\operatorname{six}^{5}$ and $\sqrt{3}$.

We consider bases of constants by including multiple polylogarithm values at sixth roots of unity up to weight six, i.e. of the form $G\left(a_{1}, \ldots, a_{w} ; 1\right)$ where the indices $a_{i}$ are equal to zero or a sixth root of unity, i.e. taken from the seven-letters alphabet $\left\{0, r_{1}, r_{3},-1, r_{4}, r_{2}, 1\right\}$ with

$$
r_{1,2}=\frac{1}{2}(1 \pm \sqrt{3} \mathrm{i})=\lambda^{ \pm 1}, \quad r_{3,4}=\frac{1}{2}(-1 \pm \sqrt{3} \mathrm{i})=\lambda^{ \pm 2}, \quad \lambda=e^{\pi \mathrm{i} / 3}=r_{1} .
$$

and $a_{1} \neq 1$.

The multiple polylogarithms are defined as

$$
G\left(a_{1}, \ldots, a_{w} ; z\right)=\int_{0}^{z} \frac{1}{t-a_{1}} G\left(a_{2}, \ldots, a_{w} ; t\right) \mathrm{d} t
$$

with $a_{i}, z \in \mathbb{C}$ and $G(z)=1$. In the special case where $a_{i}=0$ for all $i$, the corresponding integral is divergent and instead one defines

$$
G(0, \ldots, 0 ; z)=\frac{1}{n !} \log ^{n} z
$$

\footnotetext{
${ }^{5}$ Bases up to weight three were constructed in ref. [60] and up to weight four in ref. [61].
} 
If $a_{w} \neq 0$ and $\rho \neq 0$, then $G\left(\rho a_{1}, \ldots, \rho a_{w} ; \rho z\right)=G\left(a_{1}, \ldots, a_{w} ; z\right)$ so that one can express such MPL in terms of $G(\ldots ; 1)$. The length $w$ of the index vector is called the weight. One can consider separately the real and imaginary parts of the MPL

$$
G\left(a_{1}, \ldots, a_{w} ; 1\right)=G_{R}\left(a_{1}, \ldots, a_{w}\right)+\mathrm{i} G_{I}\left(a_{1}, \ldots, a_{w}\right)
$$

For example, the elements of weight one are chosen in ref. [35] as

$$
\begin{aligned}
G_{R}(-1) & =\log (2), \\
G_{R}\left(r_{4}\right) & =\frac{1}{2} \log (3), \\
G_{I}\left(r_{2}\right) & =-\frac{\pi}{3} .
\end{aligned}
$$

Let us denote by $B_{R}(w)\left(B_{I}(w)\right)$ the bases generated by $G_{R}\left(a_{1}, \ldots, a_{w}\right)\left(G_{I}\left(a_{1}, \ldots, a_{w}\right)\right)$. They include not only elements of the form $G_{R / I}\left(a_{1}, \ldots, a_{w}\right)$ but also products of constants of lower weights. The definitions of the bases can also be found in auxiliary files supplied with ref. [35]. They can be downloaded from http://theory.sinp.msu.ru/ smirnov/mpl6.tar.gz.

As we shall see in our case in practice, when using the PSLQ algorithm, it is sufficient to use the bases $B(w)=\left\{B_{R}(w), \sqrt{3} B_{I}(w)\right\}$ of weights $w=1,2, \ldots$ The element $\sqrt{3}$ does not contribute to the weight, so that elements from $\sqrt{3} B_{I}(w)$ are 'real'. To get rid of $\sqrt{3}$ in our results, we can turn to rescaled imaginary elements via

$$
\tilde{G}_{I}\left(a_{1}, \ldots, a_{w}\right)=\sqrt{3} G_{I}\left(a_{1}, \ldots, a_{w}\right) .
$$

The numbers of elements in these bases $B(w)$ are $3,8,21,55,144$ for weights $w=1,2,3,4,5$, correspondingly. If a constant is expected to be uniformly transcendental one can use these bases. Otherwise, one uses

$$
\bar{B}(w)=\bigcup_{i=1}^{w} B(i) .
$$

The numbers of elements in these bases are 4, 12, 33, 88, 232 for weights $w=1,2,3,4,5$, correspondingly.

In simple situations, the number of available digits per constant in a basis can be as small as 7. In more complicated situations, with cumbersome coefficients in results, it can be more than 15. In our case, the accuracy of 2000 digits was quite enough to obtain results with PSLQ in an $\epsilon$-expansion up to the finite part in $\epsilon$, or, in other words, up to weight 4, in a straightforward way. Still at weight 5 , it looks like the coefficients in results are more cumbersome and it is better to simplify our approach.

Let us look for uniformly transcendental threshold integrals. An analysis of results for the corresponding on-shell integrals, i.e. at $p^{2}=m^{2}$ shows that the integrals

$$
\left\{J_{4}=F_{1,2,2,2,2,0, \ldots, 0}, J_{5}=F_{2,2,2,2,1,0, \ldots, 0}\right\} .
$$

are uniformly transcendental. Let us assume that these integrals at $p^{2}=9 \mathrm{~m}^{2}$ also have this property. To check this hypothesis, we run PSLQ on coefficients of $\epsilon$-expansions of these 
integrals, with the use of uniformly transcendental bases $B(w)$ and arrive at the following results up to the finite part in $\epsilon$ :

$$
\begin{aligned}
J_{4}= & \frac{1}{\epsilon}\left(-\frac{20}{9} \tilde{G}_{I}\left(r_{2}\right) \tilde{G}_{I}\left(0, r_{2}\right)-\frac{26}{9} G_{R}(0,0,1)\right) \\
& -\frac{16}{3} \tilde{G}_{I}\left(r_{2}\right) G_{R}\left(r_{4}\right) \tilde{G}_{I}\left(0, r_{2}\right)+\frac{124}{3} \tilde{G}_{I}\left(r_{2}\right) \tilde{G}_{I}\left(0,1, r_{4}\right) \\
& +24 \tilde{G}_{I}\left(r_{2}\right) \tilde{G}_{I}\left(0, r_{2},-1\right)-\frac{100}{9} \tilde{G}_{I}\left(0, r_{2}\right)^{2}+8 G_{R}\left(0,0, r_{4}, 1\right)+\frac{1153}{135} \tilde{G}_{I}\left(r_{2}\right)^{4}+O(\epsilon),
\end{aligned}
$$

and

$$
\begin{aligned}
J_{5}= & \frac{\tilde{G}_{I}\left(r_{2}\right)}{18 \epsilon^{3}}+\frac{1}{\epsilon^{2}}\left(\frac{5}{9} \tilde{G}_{I}\left(0, r_{2}\right)-\frac{5}{9} \tilde{G}_{I}\left(r_{2}\right) G_{R}\left(r_{4}\right)-G_{R}(-1) \tilde{G}_{I}\left(r_{2}\right)\right) \\
& +\frac{1}{\epsilon}\left(-\frac{52}{9} G_{R}\left(r_{4}\right) \tilde{G}_{I}\left(0, r_{2}\right)-10 G_{R}(-1) \tilde{G}_{I}\left(0, r_{2}\right)+\frac{40}{27} \tilde{G}_{I}\left(r_{2}\right) \tilde{G}_{I}\left(0, r_{2}\right)+6 \tilde{G}_{I}\left(0, r_{2},-1\right)\right. \\
& +\frac{26}{3} \tilde{G}_{I}\left(0,1, r_{4}\right)+\frac{52}{27} G_{R}(0,0,1)+\frac{25}{9} \tilde{G}_{I}\left(r_{2}\right) G_{R}\left(r_{4}\right)^{2}+10 G_{R}(-1) \tilde{G}_{I}\left(r_{2}\right) G_{R}\left(r_{4}\right) \\
& \left.+9 G_{R}(-1)^{2} \tilde{G}_{I}\left(r_{2}\right)+\frac{253 \tilde{G}_{I}\left(r_{2}\right)^{3}}{108}\right) \\
& +\frac{32}{9} \tilde{G}_{I}\left(r_{2}\right) G_{R}\left(r_{4}\right) \tilde{G}_{I}\left(0, r_{2}\right)+\frac{1060}{27} G_{R}\left(r_{4}\right)^{2} \tilde{G}_{I}\left(0, r_{2}\right)-60 G_{R}\left(r_{4}\right) \tilde{G}_{I}\left(0, r_{2},-1\right) \\
& +104 G_{R}(-1) G_{R}\left(r_{4}\right) \tilde{G}_{I}\left(0, r_{2}\right)+\frac{5101}{324} G_{R}(0,0,1) \tilde{G}_{I}\left(r_{2}\right)+90 G_{R}(-1)^{2} \tilde{G}_{I}\left(0, r_{2}\right) \\
& -54 G_{R}(-1) \tilde{G}_{I}\left(0, r_{2},-1\right)+14 \tilde{G}_{I}\left(0, r_{2}\right) G_{R}\left(r_{2},-1\right)-96 G_{R}(-1) \tilde{G}_{I}\left(0,1, r_{4}\right) \\
& -\frac{530}{9} G_{R}\left(r_{4}\right) \tilde{G}_{I}\left(0,1, r_{4}\right)-60 \tilde{G}_{I}\left(0,1, r_{2}, r_{3}\right)-\frac{248}{9} \tilde{G}_{I}\left(r_{2}\right) \tilde{G}_{I}\left(0,1, r_{4}\right) \\
& +\frac{5695}{108} \tilde{G}_{I}\left(r_{2}\right)^{2} \tilde{G}_{I}\left(0, r_{2}\right)-16 \tilde{G}_{I}\left(r_{2}\right) \tilde{G}_{I}\left(0, r_{2},-1\right)+\frac{200}{27} \tilde{G}_{I}\left(0, r_{2}\right)^{2} \\
& -\frac{7438}{81} \tilde{G}_{I}\left(0,0,0, r_{2}\right)-74 \tilde{G}_{I}\left(0,1, r_{2},-1\right)+54 \tilde{G}_{I}\left(0, r_{2}, 1,-1\right) \\
& +\frac{250}{9} \tilde{G}_{I}\left(0,1,1, r_{4}\right)-\frac{16}{3} G_{R}\left(0,0, r_{4}, 1\right)-\frac{1021}{27} \tilde{G}_{I}\left(r_{2}\right)^{3} G_{R}\left(r_{4}\right)-\frac{250}{27} \tilde{G}_{I}\left(r_{2}\right) G_{R}\left(r_{4}\right)^{3} \\
& -50 G_{R}(-1) \tilde{G}_{I}\left(r_{2}\right) G_{R}\left(r_{4}\right)^{2}-90 G_{R}(-1)^{2} \tilde{G}_{I}\left(r_{2}\right) G_{R}\left(r_{4}\right)-\frac{287}{6} G_{R}(-1) \tilde{G}_{I}\left(r_{2}\right)^{3} \\
& -54 G_{R}(-1)^{3} \tilde{G}_{I}\left(r_{2}\right)-\frac{2306}{405} \tilde{G}_{I}\left(r_{2}\right)^{4}+O(\epsilon) . \\
& -9 v i t
\end{aligned}
$$

To evaluate the $\epsilon$-term of the first threshold master integral $J_{1}$ (considered at the point $x=9$ ) let us construct the following linear combination:

$$
J_{6}=\left(1+\frac{1}{2} \epsilon+\frac{95}{12} \epsilon^{2}+\frac{2615}{144} \epsilon^{3}+\frac{1154333}{1728} \epsilon^{4}\right) J_{1}+48 \epsilon J_{4}-3024 \epsilon^{3} J_{5} .
$$

The coefficients here are adjusted in such a way that the available result for $J_{6}$ is uniformly transcendental up to the finite part in $\epsilon$. Moreover, analytical result for its $\epsilon$-term can be revealed with the help of the basis

$$
\tilde{B}(5)=B(5) \cup\left\{1, \tilde{G}_{I}\left(r_{2}\right),-\frac{20}{9} \tilde{G}_{I}\left(r_{2}\right) \tilde{G}_{I}\left(0, r_{2}\right)-\frac{26}{9} G_{R}(0,0,1)\right\} .
$$


which differs from the uniformly transcendental basis of weight 5 given by eq. (3.8) by adding three elements that are proportional to the leading terms of $J_{1}, J_{5}, J_{4}$ in their $\epsilon$-expansions. One therefore can expect that the construction of the $\epsilon$-expansion of the coefficients in front of $J_{1}, J_{4}$, and $J_{5}$ in eq. (3.10) can be continued in such a way as to provide uniform transcendentality of $J_{6}$ up to any given order in $\epsilon$. Then one can even try to guess the exact rational coefficients in the uniformly transcendental combination of $J_{1,4,5}$ by using a Padé approximation. This is exactly the procedure that was used, e.g., in ref. [62] to discover uniformly transcendental integrals. However, for our present result the order of $\epsilon$-expansion of the coefficients is not sufficient to perform this trick. As to the question whether the same combinations will remain uniformly transcendental at arbitrary $s$, we can cautiously suggest that this will, indeed, be the case provided that a proper notion of transcendentality is elaborated for the integrals beyond polylogarithms.

Running PSLQ on a high-precision numerical value of the $\epsilon$-term of $J_{6}$, with this basis we obtain an analytical result from which we derive the $\epsilon$-term of $J_{1}$ and thereby arrive at the following result for the first master integral at threshold

$$
\begin{aligned}
J_{1}= & -\frac{1}{4 \epsilon^{4}}+\frac{1}{8 \epsilon^{3}}+\frac{1}{\epsilon^{2}}\left(\frac{23}{12}-\frac{\tilde{G}_{I}\left(r_{2}\right)^{2}}{4}\right)+\frac{1}{\epsilon}\left(-\frac{1}{3} G_{R}(0,0,1)+\frac{\tilde{G}_{I}\left(r_{2}\right)^{2}}{8}+\frac{1493}{576}\right) \\
& -40 \tilde{G}_{I}\left(r_{2}\right) G_{R}\left(r_{4}\right) \tilde{G}_{I}\left(0, r_{2}\right)+60 \tilde{G}_{I}\left(r_{2}\right) \tilde{G}_{I}\left(0,1, r_{4}\right)+\frac{320}{3} \tilde{G}_{I}\left(r_{2}\right) \tilde{G}_{I}\left(0, r_{2}\right) \\
& +72 G_{R}\left(0,0, r_{4}, 1\right)+\frac{833}{6} G_{R}(0,0,1)+\frac{647 \tilde{G}_{I}\left(r_{2}\right)^{4}}{60}+\frac{23 \tilde{G}_{I}\left(r_{2}\right)^{2}}{12} \\
& +168 \tilde{G}_{I}\left(r_{2}\right)+\frac{1024805}{6912} \\
& +\epsilon\left(-352 \tilde{G}_{I}\left(r_{2}\right) G_{R}\left(r_{4}\right)^{2} \tilde{G}_{I}\left(0, r_{2}\right)-864 G_{R}(-1) \tilde{G}_{I}\left(r_{2}\right) \tilde{G}_{I}\left(0,1, r_{4}\right)\right. \\
& +276 \tilde{G}_{I}\left(r_{2}\right) G_{R}\left(r_{4}\right) \tilde{G}_{I}\left(0, r_{2}\right)+528 \tilde{G}_{I}\left(r_{2}\right) G_{R}\left(r_{4}\right) \tilde{G}_{I}\left(0,1, r_{4}\right) \\
& +864 \tilde{G}_{I}\left(r_{2}\right) G_{R}\left(r_{4}\right) \tilde{G}_{I}\left(0, r_{2},-1\right)-\frac{15563}{27} G_{R}(0,0,1) \tilde{G}_{I}\left(r_{2}\right)^{2} \\
& +576 \tilde{G}_{I}\left(r_{2}\right) \tilde{G}_{I}\left(0, r_{2}\right) G_{R}\left(r_{2},-1\right)+864 \tilde{G}_{I}\left(r_{2}\right) \tilde{G}_{I}\left(0,1, r_{2}, r_{3}\right)-2014 \tilde{G}_{I}\left(r_{2}\right) \tilde{G}_{I}\left(0,1, r_{4}\right) \\
& -960 \tilde{G}_{I}\left(r_{2}\right) \tilde{G}_{I}\left(0,1,1, r_{4}\right)+568 \tilde{G}_{I}\left(0, r_{2}\right) \tilde{G}_{I}\left(0,1, r_{4}\right)-\frac{72172}{81} \tilde{G}_{I}\left(r_{2}\right)^{3} \tilde{G}_{I}\left(0, r_{2}\right) \\
& +\frac{320}{27} \tilde{G}_{I}\left(r_{2}\right) \tilde{G}_{I}\left(0, r_{2}\right)-1152 \tilde{G}_{I}\left(r_{2}\right) \tilde{G}_{I}\left(0, r_{2},-1\right)+\frac{14816}{9} \tilde{G}_{I}\left(r_{2}\right) \tilde{G}_{I}\left(0,0,0, r_{2}\right) \\
& +288 \tilde{G}_{I}\left(r_{2}\right) \tilde{G}_{I}\left(0,1, r_{2},-1\right)+\frac{1600}{3} \tilde{G}_{I}\left(0, r_{2}\right)^{2}+1680 \tilde{G}_{I}\left(0, r_{2}\right) \\
& +1136 G_{R}\left(0,0,1, r_{2}, r_{4}\right)+288 G_{R}\left(r_{4}\right) G_{R}\left(0,0, r_{4}, 1\right)-420 G_{R}\left(0,0, r_{4}, 1\right) \\
& -288 G_{R}\left(0,0,1,1, r_{4}\right)+\frac{485}{27} G_{R}(0,0,1)-\frac{397811}{405} G_{R}(0,0,0,0,1) \\
& +\frac{5132}{15} \tilde{G}_{I}\left(r_{2}\right)^{4} G_{R}\left(r_{4}\right)-1680 \tilde{G}_{I}\left(r_{2}\right) G_{R}\left(r_{4}\right)+168 G_{R}(-1) \tilde{G}_{I}\left(r_{2}\right)^{4} \\
& -3024 G_{R}(-1) \tilde{G}_{I}\left(r_{2}\right)-\frac{29905 \tilde{G}_{I}\left(r_{2}\right)^{4}}{72}+\frac{1493 \tilde{G}_{I}\left(r_{2}\right)^{2}}{576} \\
& \left.\frac{27244 \tilde{G}_{I}\left(r_{2}\right)}{9}+\frac{232538063}{82944}\right)+O\left(\epsilon^{2}\right) . \\
& \\
&
\end{aligned}
$$


We apply this procedure based on uniformly transcendental bases also to $J_{2}$ and $J_{3}$. Here we use, in a similar way, the following two linear combinations of the threshold master integrals and adjust coefficients in such a way that the available results up to the finite part in $\epsilon$ (but not to higher orders of expansion) are uniformly transcendental

$$
\begin{aligned}
& J_{7}=\left(1+\frac{1}{3} \epsilon+\frac{37}{9} \epsilon^{2}+\frac{571}{108} \epsilon^{3}+\frac{139585}{324} \epsilon^{4}\right) J_{2}-37 \epsilon J_{4}+2112 \epsilon^{3} J_{5}, \\
& J_{8}=\left(1+8 \epsilon^{2}-\frac{277}{2} \epsilon^{3}-\frac{29551}{12} \epsilon^{4}\right) J_{3}+8(6 \epsilon-1) J_{4}+16(743 \epsilon+48) \epsilon^{2} J_{5} .
\end{aligned}
$$

Then for the second and the third master integrals at threshold, we obtain the following results up to $\epsilon^{1}$ :

$$
\begin{aligned}
& J_{2}=-\frac{1}{4 \epsilon^{4}}+\frac{1}{\epsilon^{2}}\left(2-\frac{\tilde{G}_{I}\left(r_{2}\right)^{2}}{4}\right) \\
& +\frac{1}{\epsilon}\left(-\frac{160}{9} \tilde{G}_{I}\left(r_{2}\right) \tilde{G}_{I}\left(0, r_{2}\right)-\frac{211}{9} G_{R}(0,0,1)-\frac{128 \tilde{G}_{I}\left(r_{2}\right)}{3}-\frac{277}{8}\right) \\
& +40 \tilde{G}_{I}\left(r_{2}\right) G_{R}\left(r_{4}\right) \tilde{G}_{I}\left(0, r_{2}\right)-60 \tilde{G}_{I}\left(r_{2}\right) \tilde{G}_{I}\left(0,1, r_{4}\right)-\frac{740}{9} \tilde{G}_{I}\left(r_{2}\right) \tilde{G}_{I}\left(0, r_{2}\right) \\
& -72 G_{R}\left(0,0, r_{4}, 1\right)-107 G_{R}(0,0,1)-\frac{647}{60} \tilde{G}_{I}\left(r_{2}\right)^{4}-\tilde{G}_{I}\left(r_{2}\right)^{2}-\frac{352 \tilde{G}_{I}\left(r_{2}\right)}{3}-\frac{1647}{16} \\
& +\epsilon\left(352 \tilde{G}_{I}\left(r_{2}\right) G_{R}\left(r_{4}\right)^{2} \tilde{G}_{I}\left(0, r_{2}\right)+864 G_{R}(-1) \tilde{G}_{I}\left(r_{2}\right) \tilde{G}_{I}\left(0,1, r_{4}\right)\right. \\
& -\frac{632}{3} \tilde{G}_{I}\left(r_{2}\right) G_{R}\left(r_{4}\right) \tilde{G}_{I}\left(0, r_{2}\right)-528 \tilde{G}_{I}\left(r_{2}\right) G_{R}\left(r_{4}\right) \tilde{G}_{I}\left(0,1, r_{4}\right) \\
& -864 \tilde{G}_{I}\left(r_{2}\right) G_{R}\left(r_{4}\right) \tilde{G}_{I}\left(0, r_{2},-1\right)+\frac{15563}{27} G_{R}(0,0,1) \tilde{G}_{I}\left(r_{2}\right)^{2} \\
& -576 \tilde{G}_{I}\left(r_{2}\right) \tilde{G}_{I}\left(0, r_{2}\right) G_{R}\left(r_{2},-1\right)-864 \tilde{G}_{I}\left(r_{2}\right) \tilde{G}_{I}\left(0,1, r_{2}, r_{3}\right) \\
& +\frac{4648}{3} \tilde{G}_{I}\left(r_{2}\right) \tilde{G}_{I}\left(0,1, r_{4}\right)+960 \tilde{G}_{I}\left(r_{2}\right) \tilde{G}_{I}\left(0,1,1, r_{4}\right)-568 \tilde{G}_{I}\left(0, r_{2}\right) \tilde{G}_{I}\left(0,1, r_{4}\right) \\
& +\frac{72172}{81} \tilde{G}_{I}\left(r_{2}\right)^{3} \tilde{G}_{I}\left(0, r_{2}\right)+\frac{3310}{81} \tilde{G}_{I}\left(r_{2}\right) \tilde{G}_{I}\left(0, r_{2}\right)+888 \tilde{G}_{I}\left(r_{2}\right) \tilde{G}_{I}\left(0, r_{2},-1\right) \\
& -\frac{14816}{9} \tilde{G}_{I}\left(r_{2}\right) \tilde{G}_{I}\left(0,0,0, r_{2}\right)-288 \tilde{G}_{I}\left(r_{2}\right) \tilde{G}_{I}\left(0,1, r_{2},-1\right)-\frac{3700}{9} \tilde{G}_{I}\left(0, r_{2}\right)^{2} \\
& -\frac{3520}{3} \tilde{G}_{I}\left(0, r_{2}\right)-1136 G_{R}\left(0,0,1, r_{2}, r_{4}\right)-288 G_{R}\left(r_{4}\right) G_{R}\left(0,0, r_{4}, 1\right) \\
& +320 G_{R}\left(0,0, r_{4}, 1\right)+288 G_{R}\left(0,0,1,1, r_{4}\right)+\frac{4195}{81} G_{R}(0,0,1) \\
& +\frac{397811}{405} G_{R}(0,0,0,0,1)-\frac{5132}{15} \tilde{G}_{I}\left(r_{2}\right)^{4} G_{R}\left(r_{4}\right)+\frac{3520}{3} \tilde{G}_{I}\left(r_{2}\right) G_{R}\left(r_{4}\right) \\
& -168 G_{R}(-1) \tilde{G}_{I}\left(r_{2}\right)^{4}+2112 G_{R}(-1) \tilde{G}_{I}\left(r_{2}\right)+\frac{34517 \tilde{G}_{I}\left(r_{2}\right)^{4}}{108} \\
& \left.-\frac{31 \tilde{G}_{I}\left(r_{2}\right)^{2}}{48}-\frac{53122 \tilde{G}_{I}\left(r_{2}\right)}{27}-\frac{1046989}{576}\right)+O\left(\epsilon^{2}\right),
\end{aligned}
$$




$$
\begin{aligned}
& J_{3}=-\frac{1}{4 \epsilon^{4}}+\frac{1}{\epsilon^{2}}\left(2-\frac{\tilde{G}_{I}\left(r_{2}\right)^{2}}{4}\right) \\
& +\frac{1}{\epsilon}\left(-\frac{160}{9} \tilde{G}_{I}\left(r_{2}\right) \tilde{G}_{I}\left(0, r_{2}\right)-\frac{211}{9} G_{R}(0,0,1)-\frac{128 \tilde{G}_{I}\left(r_{2}\right)}{3}-\frac{277}{8}\right) \\
& -\frac{248}{3} \tilde{G}_{I}\left(r_{2}\right) G_{R}\left(r_{4}\right) \tilde{G}_{I}\left(0, r_{2}\right)+\frac{1172}{3} \tilde{G}_{I}\left(r_{2}\right) \tilde{G}_{I}\left(0,1, r_{4}\right)+\frac{320}{3} \tilde{G}_{I}\left(r_{2}\right) \tilde{G}_{I}\left(0, r_{2}\right) \\
& +192 \tilde{G}_{I}\left(r_{2}\right) \tilde{G}_{I}\left(0, r_{2},-1\right)-\frac{800}{9} \tilde{G}_{I}\left(0, r_{2}\right)^{2}-\frac{1280}{3} \tilde{G}_{I}\left(0, r_{2}\right)+136 G_{R}\left(0,0, r_{4}, 1\right) \\
& +\frac{416}{3} G_{R}(0,0,1)+\frac{1280}{3} \tilde{G}_{I}\left(r_{2}\right) G_{R}\left(r_{4}\right)+768 G_{R}(-1) \tilde{G}_{I}\left(r_{2}\right)+\frac{42719 \tilde{G}_{I}\left(r_{2}\right)^{4}}{540} \\
& +2 \tilde{G}_{I}\left(r_{2}\right)^{2}-\frac{5944 \tilde{G}_{I}\left(r_{2}\right)}{9}-\frac{30319}{48} \\
& +\epsilon\left(-\frac{25952}{27} \tilde{G}_{I}\left(r_{2}\right) G_{R}\left(r_{4}\right)^{2} \tilde{G}_{I}\left(0, r_{2}\right)-3552 G_{R}(-1) \tilde{G}_{I}\left(r_{2}\right) \tilde{G}_{I}\left(0,1, r_{4}\right)\right. \\
& +256 \tilde{G}_{I}\left(r_{2}\right) G_{R}\left(r_{4}\right) \tilde{G}_{I}\left(0, r_{2}\right)+\frac{976}{9} \tilde{G}_{I}\left(r_{2}\right) G_{R}\left(r_{4}\right) \tilde{G}_{I}\left(0,1, r_{4}\right) \\
& +1632 \tilde{G}_{I}\left(r_{2}\right) G_{R}\left(r_{4}\right) \tilde{G}_{I}\left(0, r_{2},-1\right)+\frac{16640}{9} G_{R}\left(r_{4}\right) \tilde{G}_{I}\left(0, r_{2}\right)^{2}+\frac{13312}{3} G_{R}\left(r_{4}\right) \tilde{G}_{I}\left(0, r_{2}\right) \\
& +\frac{53165}{243} G_{R}(0,0,1) \tilde{G}_{I}\left(r_{2}\right)^{2}-1728 G_{R}(-1) \tilde{G}_{I}\left(r_{2}\right) \tilde{G}_{I}\left(0, r_{2},-1\right) \\
& +640 \tilde{G}_{I}\left(r_{2}\right) \tilde{G}_{I}\left(0, r_{2}\right) G_{R}\left(r_{2},-1\right)+7680 G_{R}(-1) \tilde{G}_{I}\left(0, r_{2}\right)+3552 \tilde{G}_{I}\left(r_{2}\right) \tilde{G}_{I}\left(0,1, r_{2}, r_{3}\right) \\
& -1984 \tilde{G}_{I}\left(r_{2}\right) \tilde{G}_{I}\left(0,1, r_{4}\right)-\frac{24320}{9} \tilde{G}_{I}\left(r_{2}\right) \tilde{G}_{I}\left(0,1,1, r_{4}\right)+\frac{34616}{9} \tilde{G}_{I}\left(0, r_{2}\right) \tilde{G}_{I}\left(0,1, r_{4}\right) \\
& -\frac{2392484}{729} \tilde{G}_{I}\left(r_{2}\right)^{3} \tilde{G}_{I}\left(0, r_{2}\right)+\frac{1120}{9} \tilde{G}_{I}\left(r_{2}\right) \tilde{G}_{I}\left(0, r_{2}\right)-1152 \tilde{G}_{I}\left(r_{2}\right) \tilde{G}_{I}\left(0, r_{2},-1\right) \\
& +\frac{146656}{27} \tilde{G}_{I}\left(r_{2}\right) \tilde{G}_{I}\left(0,0,0, r_{2}\right)+2912 \tilde{G}_{I}\left(r_{2}\right) \tilde{G}_{I}\left(0,1, r_{2},-1\right) \\
& -1728 \tilde{G}_{I}\left(r_{2}\right) \tilde{G}_{I}\left(0, r_{2}, 1,-1\right)+\frac{1600}{3} \tilde{G}_{I}\left(0, r_{2}\right)^{2}-\frac{59440}{9} \tilde{G}_{I}\left(0, r_{2}\right) \\
& +1920 \tilde{G}_{I}\left(0, r_{2}\right) \tilde{G}_{I}\left(0, r_{2},-1\right)-4608 \tilde{G}_{I}\left(0, r_{2},-1\right)-6656 \tilde{G}_{I}\left(0,1, r_{4}\right) \\
& +\frac{119152}{9} G_{R}\left(0,0,1, r_{2}, r_{4}\right)-7680 G_{R}(0,0,1) G_{R}\left(r_{2},-1\right) \\
& +11520 G_{R}(-1) G_{R}\left(0,0, r_{2},-1\right)+11520 G_{R}\left(0,0,1, r_{2},-1\right)+11520 G_{R}\left(0,0, r_{2}, 1,-1\right) \\
& +544 G_{R}\left(r_{4}\right) G_{R}\left(0,0, r_{4}, 1\right)-384 G_{R}\left(0,0, r_{4}, 1\right)-544 G_{R}\left(0,0,1,1, r_{4}\right) \\
& -8960 G_{R}(-1)^{2} G_{R}(0,0,1)+\frac{1480}{9} G_{R}(0,0,1)-\frac{33658939 G_{R}(0,0,0,0,1)}{3645} \\
& -10240 G_{R}(0,0,1,1,-1)+\frac{250204}{135} \tilde{G}_{I}\left(r_{2}\right)^{4} G_{R}\left(r_{4}\right)-\frac{6400}{3} \tilde{G}_{I}\left(r_{2}\right) G_{R}\left(r_{4}\right)^{2} \\
& -7680 G_{R}(-1) \tilde{G}_{I}\left(r_{2}\right) G_{R}\left(r_{4}\right)+\frac{59440}{9} \tilde{G}_{I}\left(r_{2}\right) G_{R}\left(r_{4}\right)-\frac{1480}{3} G_{R}(-1) \tilde{G}_{I}\left(r_{2}\right)^{4} \\
& -6912 G_{R}(-1)^{2} \tilde{G}_{I}\left(r_{2}\right)+11888 G_{R}(-1) \tilde{G}_{I}\left(r_{2}\right)-\frac{18448 \tilde{G}_{I}\left(r_{2}\right)^{4}}{45}-\frac{16192 \tilde{G}_{I}\left(r_{2}\right)^{3}}{9} \\
& \left.-\frac{277 \tilde{G}_{I}\left(r_{2}\right)^{2}}{8}-\frac{170156 \tilde{G}_{I}\left(r_{2}\right)}{27}-\frac{1763005}{288}\right)+O\left(\epsilon^{2}\right) \text {. }
\end{aligned}
$$




\section{Conclusion}

Using our algorithm to solve differential equations by expansions near singular points we obtained high-precision values for our master integrals at threshold and then arrived, with the use of the PSLQ algorithm, at analytical values. In other words, with our procedure we have transported simple information about the master integrals in the large-momentum limit to the complicated point $p^{2}=9 \mathrm{~m}^{2}$ and obtained there analytical results. Moreover, starting from our boundary conditions at infinity, we analyzed not only the 'naive' part of threshold expansion but also leading terms of the form $(9-x)^{n-6 \epsilon}(n$ is integer) and observed that the same bases also work and lead to analytical results via the PSLQ algorithm. Besides, proceeding in a similar way we arrived at the (Taylor) expansions at the singular point $x=0$, with coefficients in terms of elements of our bases, in agreement with results for vacuum integrals $[63,64]$. Therefore, we have demonstrated that although a canonical form of differential equations in our example is impossible and we don't know analytical results for the integrals, we can obtain analytical results for these integrals at some special kinematic points where the integrals are expressed in terms of usual polylogarithmic constants.

We have obtained results up to $\epsilon^{1}$ but we believe that multiple polylogarithms values at sixth roots of unity form bases also at higher weights. The only possible complication when going to higher orders is connected with the fact that the size of the bases rapidly grows with the transcendental weight. We would like to emphasize that the bottleneck here is not connected with obtaining high-precision results using DESS, but rather with subsequent applications of the PSLQ algorithm. In fact, after the current calculation was done, we realized that we might use smaller (by 20-25 percents) bases defined in ref. [39] via values of harmonic polylogarithms at sixth roots of unity. At least, the results presented in this paper can be expressed also in terms of these constants. Of course, it should be simpler to try to extend these results to higher weights using these bases.

\section{Acknowledgments}

V.S. is grateful to Michail Kalmykov and Oleg Veretin for instructive discussions. The work of A.S. and V.S. was supported by RFBR, grant 17-02-00175A. The work of R.L. was supported by the grant of the 'Basis' foundation for theoretical physics.

Open Access. This article is distributed under the terms of the Creative Commons Attribution License (CC-BY 4.0), which permits any use, distribution and reproduction in any medium, provided the original author(s) and source are credited.

\section{References}

[1] E. Remiddi and J.A.M. Vermaseren, Harmonic polylogarithms, Int. J. Mod. Phys. A 15 (2000) 725 [hep-ph/9905237] [INSPIRE].

[2] A.B. Goncharov, Multiple polylogarithms, cyclotomy and modular complexes, Math. Res. Lett. 5 (1998) 497 [arXiv:1105.2076] [INSPIRE]. 
[3] D. Maitre, HPL, a mathematica implementation of the harmonic polylogarithms, Comput. Phys. Commun. 174 (2006) 222 [hep-ph/0507152] [INSPIRE].

[4] J. Vollinga and S. Weinzierl, Numerical evaluation of multiple polylogarithms, Comput. Phys. Commun. 167 (2005) 177 [hep-ph/0410259] [INSPIRE].

[5] C.W. Bauer, A. Frink and R. Kreckel, Introduction to the GiNaC framework for symbolic computation within the C++ programming language, J. Symb. Comput. 33 (2000) 1 [cs/0004015] [INSPIRE].

[6] A.V. Kotikov, Differential equations method: New technique for massive Feynman diagrams calculation, Phys. Lett. B 254 (1991) 158 [inSPIRE].

[7] A.V. Kotikov, Differential equation method: The Calculation of $N$ point Feynman diagrams, Phys. Lett. B 267 (1991) 123 [Erratum ibid. B 295 (1992) 409] [INSPIRE].

[8] E. Remiddi, Differential equations for Feynman graph amplitudes, Nuovo Cim. A 110 (1997) 1435 [hep-th/9711188] [INSPIRE].

[9] T. Gehrmann and E. Remiddi, Differential equations for two loop four point functions, Nucl. Phys. B 580 (2000) 485 [hep-ph/9912329] [INSPIRE].

[10] T. Gehrmann and E. Remiddi, Two loop master integrals for $\gamma^{*} \rightarrow 3$ jets: The Planar topologies, Nucl. Phys. B 601 (2001) 248 [hep-ph/0008287] [INSPIRE].

[11] T. Gehrmann and E. Remiddi, Two loop master integrals for $\gamma^{*} \rightarrow 3$ jets: The Nonplanar topologies, Nucl. Phys. B 601 (2001) 287 [hep-ph/0101124] [INSPIRE].

[12] J.M. Henn, Multiloop integrals in dimensional regularization made simple, Phys. Rev. Lett. 110 (2013) 251601 [arXiv:1304.1806] [INSPIRE].

[13] R.N. Lee, Reducing differential equations for multiloop master integrals, JHEP 04 (2015) 108 [arXiv: 1411.0911] [INSPIRE].

[14] O. Gituliar and V. Magerya, Fuchsia and master integrals for splitting functions from differential equations in QCD, PoS(LL2016)030 [arXiv: 1607.00759] [INSPIRE].

[15] O. Gituliar and V. Magerya, Fuchsia: a tool for reducing differential equations for Feynman master integrals to epsilon form, Comput. Phys. Commun. 219 (2017) 329 [arXiv: 1701.04269] [INSPIRE].

[16] M. Prausa, epsilon: A tool to find a canonical basis of master integrals, Comput. Phys. Commun. 219 (2017) 361 [arXiv:1701.00725] [INSPIRE].

[17] C. Meyer, Transforming differential equations of multi-loop Feynman integrals into canonical form, JHEP 04 (2017) 006 [arXiv: 1611.01087] [INSPIRE].

[18] C. Meyer, Algorithmic transformation of multi-loop master integrals to a canonical basis with CANONICA, Comput. Phys. Commun. 222 (2018) 295 [arXiv:1705.06252] [INSPIRE].

[19] R.N. Lee and A.A. Pomeransky, Normalized Fuchsian form on Riemann sphere and differential equations for multiloop integrals, arXiv:1707.07856 [INSPIRE].

[20] U. Aglietti, R. Bonciani, L. Grassi and E. Remiddi, The Two loop crossed ladder vertex diagram with two massive exchanges, Nucl. Phys. B 789 (2008) 45 [arXiv:0705.2616] [INSPIRE].

[21] R. Bonciani, V. Del Duca, H. Frellesvig, J.M. Henn, F. Moriello and V.A. Smirnov, Two-loop planar master integrals for Higgs $\rightarrow 3$ partons with full heavy-quark mass dependence, JHEP 12 (2016) 096 [arXiv:1609.06685] [INSPIRE]. 
[22] A. Primo and L. Tancredi, On the maximal cut of Feynman integrals and the solution of their differential equations, Nucl. Phys. B 916 (2017) 94 [arXiv:1610.08397] [InSPIRE].

[23] A. Primo and L. Tancredi, Maximal cuts and differential equations for Feynman integrals. An application to the three-loop massive banana graph, Nucl. Phys. B 921 (2017) 316 [arXiv: 1704.05465] [INSPIRE].

[24] L. Adams, C. Bogner, A. Schweitzer and S. Weinzierl, The kite integral to all orders in terms of elliptic polylogarithms, J. Math. Phys. 57 (2016) 122302 [arXiv:1607.01571] [INSPIRE].

[25] L. Adams and S. Weinzierl, Feynman integrals and iterated integrals of modular forms, Commun. Num. Theor. Phys. 12 (2018) 193 [arXiv:1704.08895] [InSPIRE].

[26] L. Adams and S. Weinzierl, The $\varepsilon$-form of the differential equations for Feynman integrals in the elliptic case, Phys. Lett. B 781 (2018) 270 [arXiv:1802.05020] [INSPIRE].

[27] E. Remiddi and L. Tancredi, An Elliptic Generalization of Multiple Polylogarithms, Nucl. Phys. B 925 (2017) 212 [arXiv:1709.03622] [InSPIRE].

[28] J. Ablinger et al., Iterated Elliptic and Hypergeometric Integrals for Feynman Diagrams, J. Math. Phys. 59 (2018) 062305 [arXiv: 1706.01299] [INSPIRE].

[29] M. Hidding and F. Moriello, All orders structure and efficient computation of linearly reducible elliptic Feynman integrals, arXiv:1712.04441 [INSPIRE].

[30] J. Broedel, C. Duhr, F. Dulat and L. Tancredi, Elliptic polylogarithms and iterated integrals on elliptic curves. Part I: general formalism, JHEP 05 (2018) 093 [arXiv: 1712.07089] [INSPIRE].

[31] J. Broedel, C. Duhr, F. Dulat and L. Tancredi, Elliptic polylogarithms and iterated integrals on elliptic curves II: an application to the sunrise integral, Phys. Rev. D 97 (2018) 116009 [arXiv: 1712.07095] [INSPIRE].

[32] J. Broedel, C. Duhr, F. Dulat, B. Penante and L. Tancredi, Elliptic symbol calculus: from elliptic polylogarithms to iterated integrals of Eisenstein series, arXiv:1803.10256 [INSPIRE].

[33] R.N. Lee, A.V. Smirnov and V.A. Smirnov, Solving differential equations for Feynman integrals by expansions near singular points, JHEP 03 (2018) 008 [arXiv: 1709.07525] [INSPIRE].

[34] H.R.P. Ferguson, D.H. Bailey and S. Arno, Analysis of PSLQ, an integer relation finding algorithm, Math. Comput. 68 (1999) 351.

[35] J.M. Henn, A.V. Smirnov and V.A. Smirnov, Evaluating Multiple Polylogarithm Values at Sixth Roots of Unity up to Weight Six, Nucl. Phys. B 919 (2017) 315 [arXiv:1512.08389] [INSPIRE].

[36] M. Czakon, Tops from Light Quarks: Full Mass Dependence at Two-Loops in QCD, Phys. Lett. B 664 (2008) 307 [arXiv:0803.1400] [InSPIRE].

[37] P. Bärnreuther, M. Czakon and P. Fiedler, Virtual amplitudes and threshold behaviour of hadronic top-quark pair-production cross sections, JHEP 02 (2014) 078 [arXiv:1312.6279] [INSPIRE].

[38] S. Pozzorini and E. Remiddi, Precise numerical evaluation of the two loop sunrise graph master integrals in the equal mass case, Comput. Phys. Commun. 175 (2006) 381 [hep-ph/0505041] [INSPIRE]. 
[39] B.A. Kniehl, A.F. Pikelner and O.L. Veretin, Three-loop massive tadpoles and polylogarithms through weight six, JHEP 08 (2017) 024 [arXiv: 1705.05136] [INSPIRE].

[40] R. Mueller and D.G. Öztürk, On the computation of finite bottom-quark mass effects in Higgs boson production, JHEP 08 (2016) 055 [arXiv: 1512.08570] [INSPIRE].

[41] K. Melnikov, L. Tancredi and C. Wever, Two-loop gg $\rightarrow$ Hg amplitude mediated by a nearly massless quark, JHEP 11 (2016) 104 [arXiv:1610.03747] [inSPIRE].

[42] A.V. Smirnov, Algorithm FIRE - Feynman Integral REduction, JHEP 10 (2008) 107 [arXiv: 0807.3243] [INSPIRE].

[43] A.V. Smirnov and V.A. Smirnov, FIRE4, LiteRed and accompanying tools to solve integration by parts relations, Comput. Phys. Commun. 184 (2013) 2820 [arXiv: 1302.5885] [INSPIRE].

[44] A.V. Smirnov, FIRE5: a C++ implementation of Feynman Integral REduction, Comput. Phys. Commun. 189 (2015) 182 [arXiv:1408.2372] [INSPIRE].

[45] R.N. Lee, Presenting LiteRed: a tool for the Loop InTEgrals REDuction, arXiv:1212.2685 [INSPIRE].

[46] R.N. Lee, LiteRed 1.4: a powerful tool for reduction of multiloop integrals, J. Phys. Conf. Ser. 523 (2014) 012059 [arXiv:1310.1145] [inSPIRE].

[47] R.N. Lee and A.A. Pomeransky, Critical points and number of master integrals, JHEP 11 (2013) 165 [arXiv:1308.6676] [INSPIRE].

[48] A.V. Smirnov, FIESTA4: Optimized Feynman integral calculations with GPU support, Comput. Phys. Commun. 204 (2016) 189 [arXiv:1511.03614] [InSPIRE].

[49] P. Marquard, A.V. Smirnov, V.A. Smirnov and M. Steinhauser, Quark Mass Relations to Four-Loop Order in Perturbative QCD, Phys. Rev. Lett. 114 (2015) 142002 [arXiv: 1502.01030] [INSPIRE].

[50] P. Marquard, A.V. Smirnov, V.A. Smirnov, M. Steinhauser and D. Wellmann, $\overline{M S}$-on-shell quark mass relation up to four loops in $Q C D$ and a general $\mathrm{SU}(N)$ gauge group, Phys. Rev. D 94 (2016) 074025 [arXiv:1606.06754] [INSPIRE].

[51] K.G. Chetyrkin, Operator Expansions in the Minimal Subtraction Scheme. 1: The Gluing Method, Theor. Math. Phys. 75 (1988) 346 [InSPIRE].

[52] K.G. Chetyrkin, Operator Expansions in the Minimal Subtraction Scheme. 2: Explicit Formulas for Coefficient Functions, Theor. Math. Phys. 76 (1988) 809 [INSPIRE].

[53] V.A. Smirnov, Asymptotic expansions in limits of large momenta and masses, Commun. Math. Phys. 134 (1990) 109 [INSPIRE].

[54] V.A. Smirnov, Applied asymptotic expansions in momenta and masses, Springer Tracts Mod. Phys. 177 (2002) 1 [INSPIRE].

[55] F.A. Berends, A.I. Davydychev and N.I. Ussyukina, Threshold and pseudothreshold values of the sunset diagram, Phys. Lett. B 426 (1998) 95 [hep-ph/9712209] [INSPIRE].

[56] A.I. Davydychev and V.A. Smirnov, Threshold expansion of the sunset diagram, Nucl. Phys. B 554 (1999) 391 [hep-ph/9903328] [INSPIRE].

[57] J. Fleischer and M.Yu. Kalmykov, Single mass scale diagrams: Construction of a basis for the $\epsilon$-expansion, Phys. Lett. B 470 (1999) 168 [hep-ph/9910223] [INSPIRE]. 
[58] A.I. Davydychev and M.Yu. Kalmykov, New results for the $\epsilon$-expansion of certain one, two and three loop Feynman diagrams, Nucl. Phys. B 605 (2001) 266 [hep-th/0012189] [INSPIRE].

[59] M.Yu. Kalmykov and B.A. Kniehl, 'Sixth root of unity' and Feynman diagrams: Hypergeometric function approach point of view, Nucl. Phys. Proc. Suppl. 205-206 (2010) 129 [arXiv:1007.2373] [INSPIRE].

[60] D.J. Broadhurst, Massive three-loop Feynman diagrams reducible to $S C^{*}$ primitives of algebras of the sixth root of unity, Eur. Phys. J. C 8 (1999) 311 [hep-th/9803091] [INSPIRE].

[61] F. Moriello, Linearization and symmetrization of generalized harmonic polylogarithms, Ph.D. Thesis (2013).

[62] R.N. Lee, A.V. Smirnov and V.A. Smirnov, Master Integrals for Four-Loop Massless Propagators up to Transcendentality Weight Twelve, Nucl. Phys. B 856 (2012) 95 [arXiv: 1108.0732] [INSPIRE].

[63] M. Czakon, The Four-loop QCD $\beta$-function and anomalous dimensions, Nucl. Phys. B 710 (2005) 485 [hep-ph/0411261] [INSPIRE].

[64] Y. Schröder and A. Vuorinen, High-precision $\epsilon$-expansions of single-mass-scale four-loop vacuum bubbles, JHEP 06 (2005) 051 [hep-ph/0503209] [INSPIRE]. 